Original Research Paper

\title{
Descriptive Epidemiological, Clinical and Microbiological Features of Infective Endocarditis at a University Hospital in Saudi Arabia
}

\author{
${ }^{1,2}$ Reham Kaki and ${ }^{2,3, N a b e e l a ~ A l-A b d u l l a h ~}$ \\ ${ }^{1}$ Department of Medicine, King Abdulaziz University, Jeddah, Saudi Arabia \\ ${ }^{2}$ Department of Infection Control and Environmental Health, King Abdulaziz University Hospital, Jeddah, Saudi Arabia \\ ${ }^{3}$ Department of Public Health, College of Nursing, King Abdulaziz University, Jeddah, Saudi Arabia
}

Article history

Received: 02-01-2018

Revised: 21-04-2018

Accepted: 26-05-2018

Corresponding Author:

Reham Kaki

Department of Medicine, King Abdulaziz University, Jeddah,

Saudi Arabia

Email: rmkaki@kau.edu.sa

\begin{abstract}
Infective Endocarditis (IE) still remains a serious disease. The treatment and pathology still remain unclear. In this study, we sought to clarify the epidemiology and treatment of IE in a teaching hospital in Saudi Arabia. In this study, we sought to conduct a retrospective investigation of all adult patients who were treated for IE at our hospital and determine the characteristic demographic, clinical and pathological features. Our findings indicate that the main risk factors of IE were surgical intervention $(28 \%)$ and structural heart disease $(20 \%)$. The mean (SD) age of the patients in our study was 48 (18). The most common organisms identified were Staphylococcus aureus (40\%) and Streptococcus viridans $(30 \%)$. The most common clinical presentations were anorexia, weight loss and shortness of breath. IE was accompanied by valvular dysfunction in most cases (83\%) and vegetation (55\%). About $83 \%$ of the patients had undergone transthoracic echocardiography. Our findings provided some insight into the current trends in the epidemiology and clinical features of IE in Saudi Arabia.
\end{abstract}

Keywords: Infective Endocarditis, Treatment, Staphylococcus aureus, Streptococcus viridans, Risk Factors

\section{Introduction}

Infective Endocarditis (IE) is a life-threatening infection characterized by the inflammation of the cardiac endothelium and valvular tissue. Histopathologically, it is marked by the presence of microorganisms in the lesion. Clinically, the condition can worsen rapidly, resulting in fatality if left untreated. In fact, the mortality rate of IE continues to remain high even in the face of the considerable advances made in medical technology and pharmacology Al-Tawfiq et al. (2009). IE is most often seen in intravenous drug abusers and patients with other cardiovascular diseases, especially those who have undergone heart valve replacement AlTawfiq et al. (2009).

According to age and sex, IE is known to differ in etiological factors, clinical features, natural history and therefore treatment. Staphylococcus aureus has been identified as the most common pathogenic organism in IE in developed countries and has been the cause of severe disease in affected populations, particularly patients who are elderly and those with pre-existing anomalies Cahill et al. (2017). Clinically, the disease continues to remain challenging to diagnose and manage. One of the main reasons for the difficulty in the treatment of IE is that the affected patients generally have severe pre-existing diseases and their general condition may already be poor 3. In a recent study, Capilla et al. (2017) showed that IE continues to remain a serious condition and that $S$. aureus is the most common pathogenic organism involved in IE Capilla et al. (2017).

At present, limited data are available on the prevalence, epidemiology and etiology of IE in the Gulf region, particularly, Saudi Arabia. Other than case reports, very few studies have been published in this regard from Saudi Arabia Christine et al. (2012). Therefore, in this study, we evaluated the epidemiological, clinical and microbiological features of IE at a teaching hospital in Saudi Arabia.

\section{Methodology}

This was a retrospective study conducted on patients with IE treated at our hospital between the years 2016 and 
2017. Only adult patients diagnosed with IE were included in this study and children were excluded. The study protocol was approved by the institutional review board and need for patient consent was waived.

\section{Setting}

The study was conducted at King Abdulaziz University Hospital (KAUH), a tertiary teaching hospital affiliated to the Medical School in Jeddah, Saudi Arabia. The hospital has a capacity of 750 beds and includes all specialties. There are approximately 45, 000 patients being discharged from the hospital annually.

\section{Data Collection}

The type of study is retrospective based on patients' records were used to obtain data regarding their demographic and clinical characteristics as well as the investigative and pathological findings. The parameters taken into consideration were age, sex, nationality, clinical stage of disease as well as clinical characteristics such as signs and symptoms, presence of valvular dysfunction, vegetation, presence of large vegetation and presence of comorbidities. The records were anonymized to ensure patient confidentiality. The patient included in this study based on the diagnosis IE and we trace all cases from 2016 to 2017 .

\section{Statistical Analysis}

The retrieved data were analyzed using SPSS version 21. Comparisons of continuous variables were made and those of categorical variables were made with the chisquare test or Fisher's exact test in this study because sample size less than 50 . To assess linearity, the quadratic age effect was introduced into the model, but was not found to be statistically significant.

\section{Results}

\section{Demographic Characteristics}

During the study period, a total of 40 patients with IE were admitted to KAUH. The demographic and clinical characteristics of the patients are shown in Table 1. The average age of the patients was $48( \pm 18)$ years and IE was found to affect significantly more men than women $(75 \%$ Vs. 25\%). Non-Saudi patients (70\%) were significantly more common than Saudis. Further, acute disease was more common than sub-acute presentation of IE (53\% vs. 48\%). Medical treatment (93\%) was administered to a significantly greater number of patients as compared to surgical treatment $(P=0.06)$.

\section{Clinical Presentation}

Table 2 shows the clinical features of those enrolled in this study. The most common comorbidities among the patients were hypertension and ischemic heart disease $(30 \%$ and $36 \%$, respectively); these diseases were significantly more common than other conditions/diseases $(P=0.05)$.

For clinical manifestations, the symptom of anorexia (55\%), followed by weight loss $(50 \%)$ and shortness of breath $(48 \%)$ were significantly more common than others (Table 3). In terms of pathological findings, the most common organisms identified were $S$. aureus (40\%) followed by Streptococcus viridans (30\%). Other groups identified were Haemophilus spp., Aggregatibacter (previously Actinobacillus) spp., Cardiobacterium spp., Eikenella spp. and Kingella spp. (7.5\%) Identified as [HACEK] organisms.

Table 1: Demographic data for IE patients

\begin{tabular}{lll}
\hline Gender & Male & Female \\
\hline No $(\%)$ & $30(75)$ & $10(25)$ \\
*Age & Male & Female \\
no $( \pm \mathrm{SD})$ & $50( \pm 18)$ & $43( \pm 17)$ \\
Nationality & Saudi & Non-Saudi \\
No $(\%)$ & $12(30)$ & $28(70)$ \\
Clinical classification & Acute & Sub-acute \\
No (\%) & $21(53)$ & $19(48)$ \\
Treatment & Medical & Surgical \\
No $(\%)$ & $37(93)$ & $14(35)$ \\
$\bullet$ Fisher exact test, $P=0.06$ & & \\
\hline
\end{tabular}

Table 2: Co-morbidity associated with IE

\begin{tabular}{lll} 
Co-morbidity diseases & With disease & Without disease \\
No $=40$ & Freq $(\%)$ & $29(72)$ \\
DM & $11(28)$ & $28(70)$ \\
HTN & $12(30)$ & $28(70)$ \\
IHD & $12(36)$ & $37(92)$ \\
COPD & $3(8)$ & $40(100)$ \\
Asthma & $0(0)$ & \\
Fisher exact test, $P=0.05$ & & $\%$ \\
\hline
\end{tabular}


Reham Kaki and Nabeela Al-Abdullah / American Journal of Infectious Diseases 2018, 14 (2): 63.68 DOI: 10.3844/ajidsp.2018.63.68

Table 3: Signs and symptoms associated with IE

\begin{tabular}{lll}
\hline Signs and symptoms & With symptoms & Without symptoms \\
No $=40$ & Freq $(\%)$ & $32(80)$ \\
\hline Chills & $8(20)$ & $39(85)$ \\
Malaise & $6(15)$ & $18(45)$ \\
Anorexia & $22(55)$ & $20(50)$ \\
Weight loss & $20(50)$ & $36(87)$ \\
Arthralgia & $4(10)$ & $21(52)$ \\
Shortness of breath & $19(48)$ & $24(60)$ \\
Cough & $16(40)$ & $29(73)$ \\
Chest pain & $11(27)$ & $33(82)$ \\
Back pain & $7(18)$ & $37(93)$ \\
Petechiae & $3(7)$ & $38(95)$ \\
Roth spot & $2(5)$ & $38(75)$ \\
Clubbing & $2(5)$ & $33(82)$ \\
Splenomegaly & $7(18)$ & $29(73)$ \\
Signs of ocular neuropathy & $11(27)$ & $33(82)$ \\
Raised JVP & $7(18)$ & $40(100)$ \\
Stiff neck & $0(0)$ & $36(90)$ \\
Delirium & $4(10)$ & $37(93)$ \\
Gallop & $3(7)$ & $32(80)$ \\
Arrhythmia & $8(20)$ & \\
Fisher exact test, $P=<0.005$ & & \\
\hline
\end{tabular}

Table 4: Microorganisms isolated from IE patients

\begin{tabular}{lc}
\hline Microorganism & Freq $(\%)$ \\
\hline Staphylococcus aureus & $16(40)$ \\
Streptococcus viridans & $12(30)$ \\
Other streptococcus & $3(7.5)$ \\
Coagulase-negative Staphylococcus & $3(7.5)$ \\
HACEK & $3(7.5)$ \\
Fungi & $2(5)$ \\
Enterococcus & $1(2.5)$ \\
Total & $40(100)$ \\
\hline
\end{tabular}

Table 5: Risk factors associated with IE

\begin{tabular}{lll} 
Risk factor & Present risk & Absent risk \\
No $=40$ & Freq (\%) $(\%)$ \\
\hline Pericardia & $0(0)$ & $40(100)$ \\
IDU & $1(2)$ & $39(98)$ \\
Prosthetic heart valve & $4(10)$ & $36(90)$ \\
Structural heart disease & $9(20)$ & $31(73)$ \\
Noncompliance dental hygiene & $3(8)$ & $37(92)$ \\
Surgical intervention & $11(28)$ & $29(72)$ \\
Previous IE diagnosis & $2(5)$ & $38(95)$ \\
Hospital acquired infection & $3(9)$ & $37(92)$ \\
HD & $5(13)$ & $35(87)$ \\
Fisher exact test, $P=<0.005$ & & \\
\hline
\end{tabular}

Table 6: Complications of IE

\begin{tabular}{llc}
\hline Risk factor & Outcome & Without outcome \\
No $=40$ & Freq (\%) & $7(17)$ \\
\hline Valve dysfunction & $33(83)$ & $18(45)$ \\
Vegetation & $22(55)$ & $29(73)$ \\
Large Vegetation: more than $10 \mathrm{~mm}$ & $11(27)$ & $33(76)$ \\
Multiple & $7(17)$ & $31(77)$ \\
Ischemic or MI diseases & $9(23)$ & $36(90)$ \\
Heart block & $4(10)$ & \\
Fisher exact test, $P=<0.005$ & & \\
\hline
\end{tabular}


Table 7: Laboratory results of IE

\begin{tabular}{lll}
\hline Risk factor & With IE & Without IE \\
No=40 & Freq (\%) & Freq (\%) \\
\hline High WBC & $24(60)$ & $61(40)$ \\
Thrombocytopenia & $15(38)$ & $25(62)$ \\
High ESR level & $26(65)$ & $14(35)$ \\
High CRP level & $20(72)$ & $11(28)$ \\
High creatinine level & $19(47)$ & $21(52)$ \\
Prolonged PT & $21(53)$ & $19(47)$ \\
Prolonged PTT & $28(55)$ & $18(45)$ \\
Protein urea & $3(7)$ & $37(77)$ \\
TTE & $35(88)$ & $5(12)$ \\
TEE & $8(13)$ & $34(85)$ \\
Fisher exact test, $P=<0.005$ & & \\
\hline
\end{tabular}

The risk factors identified in this study are shown in Table 5. The most common risk factors of IE identified in this study were surgical intervention $(28 \%)$ and structural heart disease $(20 \%)$ which had a significantly higher risk than other factors. In terms of clinical manifestations, most patients exhibited valve dysfunction $(83 \%)$, followed by vegetation (55\%) (Table 6). The most common positive laboratory findings (Table 7) were elevated serum Creactive protein levels and elevated erythrocyte sedimentation rate, which were observed in $70 \%$ and $65 \%$ of the patients, respectively.

\section{Discussion}

The current study was aimed at evaluating the epidemiology, pathology and treatment of IE. Our data were based on investigations of 40 patients who have been treated during the period from 2014 to 2016. Our study is unique in that these patients have been treated at our clinics unlike other studies that have been based on the discharge diagnosis.

Long-term studies on the epidemiology of IE among adult patients with congenital heart disease have shown the use of valvular prosthetics is associated with a higher risk of IE as compared to other treatment procedures such as valve repair surgery Christine et al. (2012) .

A previous study conducted in Saudi Arabia showed that IE occurred most commonly as a result of native valve endocarditis and that the most common pathogenic organisms involved were $S$ aureus, Enterococcus faecalis and streptococci viridans Gil et al. (2017). On the other hand, the most common organisms isolated from the patients in our study were $S$. aureus $(40 \%)$, followed by $S$. viridans $(30 \%)$ (Table 4$)$. This finding is different from other studies, which have shown that $S$. viridans is the most common pathogenic organism. (Jean et al., 2017; Kuijpers et al., 2017), However, our findings are consistent with those of more recent studies (Capilla et al., 2017; Marks, et al., 2015). which have shown an increasing predominance of staphylococcal infection in some regions of the world. This change in organism together with the finding that IE occurred more often in patients with a history of pre-existing ischemic heart disease (36\%) and hypertension as well as in patients who have undergone surgery $(28 \%)$ indicate that the disease is becoming increasingly complex, which highlights the need for the specialized and dedicated approaches, as stressed by Ambrosioni et al. (2017) and Nakagawa et al. (2014) Further, the increase in staphylococcal infection is concerning since studies indicate that staphylococcal IE is associated with more serious consequences and higher mortality as compared to streptococcal IE Nakagawa et al. (2014).

The finding of men being more susceptible to IE as compared to women is consistent with the findings of previous studies in the UK and Japan (Jean et al., 2017; Kuijpers et al., 2017) However, the mean (SD) age of patients in our study was lower, 48 (18) years, than those in the abovementioned studies (52 years) (Jean et al., 2017; Kuijpers et al., 2 017), particularly, a long-term study that showed an increase in the age of IE patients over two decades Kuijpers et al. (2017). As mentioned above, the predilection for the elderly population earlier noted in a study Christine et al. (2012). from the same region as ours was not noted ours. Moreover, in our study, we found that IE affected non-Saudi patients more frequently than Saudis. However, this finding may not be significant because our hospital has a majority of nonSaudi patients since ours is the only government hospital that accepts patients of other nationalities.

Clinically, IE can vary in presentation from simple infection of the values with minimal damage to a sudden, severe, life-threatening condition. The most common signs and symptoms associated with IE are the following: chills, malaise, anorexia, weight loss, arthralgia, shortness of breath, cough and chest and back pain (Table 3). Around $55 \%$ of our patients had anorexia and $50 \%$ had weight loss, only $10 \%$ had arthralgia. The most common signs and symptoms noted in our study were anorexia, weight loss and shortness of breath. This is different from other studies that have reported more acute symptoms such as fever, chills and malaise Marks et al. (2015).

With respect to underlying disease, the most common comorbidities found in our study were 
ischemic heart disease in $36 \%$, hypertension in $30 \%$ and diabetes mellitus in $28 \%$ (Table 2). This is consistent with findings of some other studies that have shown a predominance of pre-existing heart disease (Jean et al., 2017; Kuijpers et al., 2017). Structural heart disease was present in $28 \%$ of our patients.

Our study provides an overview of the current clinical and epidemiological trends of IE in Saudi Arabia. Similar to a previous report from the country, which reported the predominance of $S$. aureus $(42.6 \%)$, ours showed that the organism was responsible for IE in $40 \%$ of the cases Christine et al. (2012). However, the percentage of $S$. viridans in our study was almost double that noted in the previous study (30\% Vs. 16.7\%) Christine et al. (2012). This suggests the need for further investigation focusing on changing patterns in the pathogenic organisms in our region, as observed in some other regions of the world. Further, the mean (SD) age of the patients in our study was much less than that reported in the previous investigation (48 (18) years Vs. 59.7 (18.2) years) (Christine et al., 2012). This could be related to the advances in healthcare facilities and increasing number of intervensive investigative and therapeutic procedures (Wan et al., 2017). Interestingly, in our study, only $10 \%$ of the patients had IE associated with the implantation of prosthetic valves. This percentage is much lower than that reported in the study by (Marks et al., 2015) (21\%) and Al-Tawfiq and Sufi (2009). (18.7\%), but closer to the study from Japan (13\%) (Wan et al., 2017). This is consistent with our finding of staphylococcal predominance since the latter are known to affect native valves, whereas streptococci have a preference for prosthetic valves Jean et al. (2017).

This study has some limitations. First, the sample population was small and this was only a single-center study. In future, we intend to investigate a larger sample population across multiple centers. Second, in this study we did not recruit a control group to compare the characteristics of patients with IE with those of patients with other cardiac diseases. Future epidemiological investigations should be used to compare the characteristics of patients with IE and other population groups. Third, we did not assess mortality in this study since this was a cross-sectional rather than a longitudinal study. We intend to undertake long-term investigations to clarify this.

To conclude, our study was able to provide some insight into the epidemiological status of IE. This disease seems to be evolving with the advances in medical technology and changes in the treatment protocol of other diseases, particularly surgical and interventive investigative procedures. Therefore, it is imperative that epidemiological studies are routinely undertaken in various parts of the world across various population groups in order to identify its peculiar clinical characteristics, risk factors and the causative pathogenic organisms and accordingly modify treatment and diagnostic procedures in order to combat IE effectively.

\section{Author's Contribution}

Nabeela Al-Abdullah: Gathered all the study information and wrote the proposal and manuscript.

Reham Kaki and Nabeela Al-Abdullah: Conceived the study, participated in the study design, performed the statistical analysis and drafted the manuscript. All authors read and approved the final manuscript.

\section{Ethics}

This article is original and contains unpublished material. The corresponding author confirms that all of the other authors have read and approved the manuscript and there are no ethical issues involved.

\section{References}

Al-Tawfiq, J.A. and I. Sufi, 2009. Infective endocarditis at a hospital in Saudi Arabia: Epidemiology, bacterial pathogens and outcome. Ann. Saudi Med., 29: 433-436. DOI: $10.4103 / 0256-4947.57164$

Ambrosioni, J., M. Hernandez-Meneses, A. Téllez, J. Pericàs and C. Falces et al., 2017. The changing epidemiology of infective endocarditis in the twentyfirst century. Curr. Infect. Dis. Rep., 19: 1-21. DOI: 10.1007/S1 1908-017-0574-9

Cahill, T.J., L.M. Baddour, G. Habib, B. Hoen and E. Salaun et al., 2017. Challenges in infective endocarditis. J. Am. College Cardiol., 69: 325-344. DOI: 10.1016/j.jacc.2016.10.066

Capilla, E., R. Poyet, A.V. Tortat, J. Marchi and F.X. Brocq et al., 2017. Infective endocarditis: clinical features and prognosis between 2004 and 2014 in a non-teaching hospital. Ann. Cardiol Angeiol, 66: 87-91. DOI: 10.1016/j.ancard.2016.09.042

Christine, S.S., C. Marie, L.M. Vincent, D.L. Thanh and C. Catherine et al., 2012. Preeminence of Staphylococcus Aureus in infective endocarditis: A 1-year populationbased survey. Clin. Infect. Dis., 54: 1230-1239. DOI: $10.1093 / \mathrm{cid} / \mathrm{cis} 199$

Gil, J., H. Antunes, B. Marmelo, L. Abreu and M.L. Goncalves et al., 2017. Septic shock of unknown origin: A case report of a pseudoaneurysm of the mitral-aortic intervalvular fibrosa. J. Clin. Med. Res., 9: 225-228. DOI: 10.14740/jocmr2903w

Jean, C.Y., N.Y. Sandra, P. Agnes, A. Parla and R. Annie et al., 2017. Staphylococcal versus Streptococcal infective endocarditis in a tertiary hospital in Belgium: epidemiology, clinical characteristics and outcome. Int. J. Clin. Laboratory Medi., 72: 417-423.

DOI: $10.1080 / 17843286.2017 .1309341$ 
Kuijpers, J.M., D.R. Koolbergen, M. Groenink, K.C. Peels and C.L. Reichert et al., 2017. Incidence, risk factors and predictors of infective endocarditis in adult congenital heart disease: Focus on the use of prosthetic material. Eur. Heart J., 38: 2048-2056. DOI: 10.1093/eurheartj/ehw591

Marks, D.J.B., C. Hyams, C.Y. Koo, M. Pavlou and J. Robbins et al., 2015. Clinical features, microbiology and surgical outcomes of infective endocarditis: A 13-year study from a uk tertiary cardiothoracic referral centre. AN Int. J. Med., 108: 219-229.

DOI: 10.1093/qjmed/hcu188
Nakagawa, T., H. Wada, K. Sakakura, Y. Yamada and K. Ishida et al., 2014. Clinical features of infective endocarditis: Comparison between the 1990s and 2000s. J. Cardiol., 63: 145-148.

DOI: 10.1016/J.JJCC.2013.06.007

Wan, Z., Z. Qian and Z. Jingping, 2017. The changing epidemiology and clinical features of infective endocarditis: A retrospective study of 196 episodes in a teaching hospital in china. BMC Cardiovascular Disorders, 17: 113-113.

DOI: $10.1186 /$ S12872-017-0548-8 